\title{
Leidenfrost Vapor Layers Reduce Drag without the Crisis in High Viscosity Liquids
}

\author{
Ivan U. Vakarelski, ${ }^{1, *}$ Joseph D. Berry, ${ }^{2,3}$ Derek Y. C. Chan, ${ }^{4,5}$ and Sigurdur T. Thoroddsen ${ }^{1}$ \\ ${ }^{1}$ Division of Physical Sciences and Engineering, King Abdullah University of Science and Technology (KAUST), \\ Thuwal 23955-6900, Saudi Arabia \\ ${ }^{2}$ Department of Chemical and Biomolecular Engineering, University of Melbourne, Parkville VIC 3010, Australia \\ ${ }^{3}$ Mineral Resources, Commonwealth Scientific and Industrial Research Organisation (CSIRO), Clayton VIC 3169, Australia \\ ${ }^{4}$ School of Mathematics and Statistics, University of Melbourne, Parkville 3010, Australia \\ ${ }^{5}$ Department of Chemistry and Biotechnology, Swinburne University of Technology, Hawthorn 3122, Australia
}

(Received 21 March 2016; published 8 September 2016)

\begin{abstract}
The drag coefficient $C_{D}$ of a solid smooth sphere moving in a fluid is known to be only a function of the Reynolds number $\operatorname{Re}$ and diminishes rapidly at the drag crisis around $\operatorname{Re} \sim 3 \times 10^{5}$. A Leidenfrost vapor layer on a hot sphere surface can trigger the onset of the drag crisis at a lower Re. By using a range of high viscosity perfluorocarbon liquids, we show that the drag reduction effect can occur over a wide range of Re, from as low as $\sim 600$ to $10^{5}$. The Navier slip model with a viscosity dependent slip length can fit the observed drag reduction and wake shape.
\end{abstract}

DOI: 10.1103/PhysRevLett.117.114503

The universal drag curve $C_{D}(\mathrm{Re})$ for a no-slip solid sphere is one of the pillars of experimental hydrodynamics. An important characteristic feature on it is the sudden fourfold reduction in the drag coefficient, $C_{D}=2 F_{D} /$ $\left(\pi R^{2} \rho U^{2}\right)$ when the Reynolds number $\operatorname{Re}=2 \rho R U / \mu$ reaches $\sim 3 \times 10^{5}$ [1]. This is the so-called drag crisis. Here, $F_{D}$ is the hydrodynamic drag force on the sphere of radius $R$ traveling with velocity $U$ in a fluid of density $\rho$ and dynamic viscosity $\mu$. Controlling the onset of the drag crisis, with dimples on a golf ball or threads on a soccer ball and baseballs, increases their range and stabilizes their trajectories, but can also save propulsive energy in maritime vessel related applications [2-4].

At speeds below the drag crisis, the boundary layer separates from the sphere at the equator and forms a large wake with low pressure at the rear of the sphere so that the higher pressure on the front side accounts for the resistive force, often referred to as the form drag. Viscous skin friction effects account for less then $5 \%$ of the drag force at high Re [1]. At speeds above the drag crisis, the point of separation moves towards the rear of the sphere and partially compensates the high front pressure, thus reducing the form drag.

The Re at which the drag crisis occurs can be lowered by roughening the surface or by engineering surface features, such as dimples on golf balls, thereby enhancing the early transition to boundary layer turbulence [2]. Other reduction methods that lower the critical Re for the onset of the drag crisis include the use of a flexible polymer dissolved in a liquid [5] and active control of the flow over the sphere [6]. Recently, it has been shown that drag reduction can arise from mass transfer resulting from the melting of an ice surface in contact with water [7]. However, all such phenomena occur within a relatively narrow range of $\operatorname{Re}, \sim 5 \times 10^{4}$ to $3 \times 10^{5}$.
In this Letter we demonstrate that a stable Leidenfrost vapor layer sustained on the surface of a free-falling sphere can dramatically reduce drag over a much wider range of Re, starting from as low as 600 to $10^{5}$. To realize this we use a range of perfluorinated liquids for which the viscosity varies by a factor of over 20 . The observed drag coefficients $C_{D}$ no longer follow a universal function of the Reynolds number. We also show that this behavior can be fitted using the Navier slip model in which the no-slip boundary condition is replaced by a phenomenological slip length that varies with the fluid viscosity.

Background.-The traditional Leidenfrost effect refers to small liquid droplets being levitated by their vapor when they are placed over a surface heated above the Leidenfrost temperature $T_{L}$, which usually is considerably higher than the liquid boiling point $T_{B}$ [8-10]. In the inverted configuration, when a solid body heated above $T_{L}$ is immersed in the liquid, it will be completely enveloped in a vapor layer (see, for example, Fig. S1 in the Supplemental Material [11]). Such vapor layers on free-falling spheres can efficiently reduce the drag and thus shift the drag crisis to a lower Re [22,23]. Such results with Leidenfrost vapor layers give an upper bound for the drag reduction possible by gas layers or plastrons, either sustained naturally on superhydrophobic surfaces or induced by microbubble injection [24-33]. Early experiments conducted using heated spheres in the perfluorocarbon liquid FC-72 comprising mainly perfuorohexane $\mathrm{C}_{6} F_{14}$ [22], as well as in water heated to $95{ }^{\circ} \mathrm{C}$ [23], appeared to suggest that the drag reduction effect of the vapor layer follows a universal dependence on Re, with deviations from the no-vapor-layer case beginning from $\operatorname{Re}>2 \times 10^{4}$ to a fully developed effect at $\mathrm{Re} \simeq 10^{5}$. Based on these earlier results one might expect that the drag reduction by vapor layers in high 


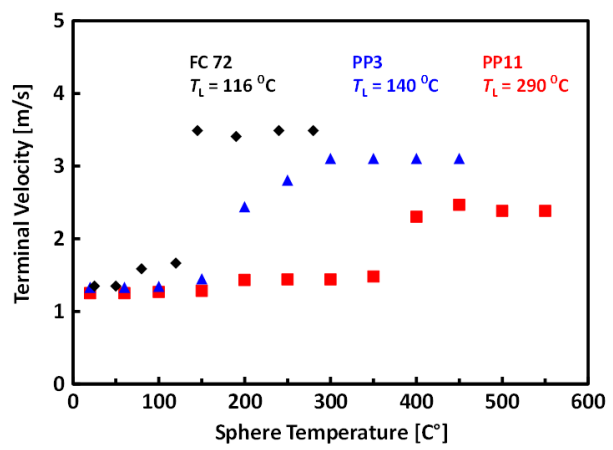

FIG. 1. Variation of the terminal velocity with the sphere temperature, measured for a $20 \mathrm{~mm}$ steel sphere falling through PP11 ( $\square$, red; $\mu=19.2 \mathrm{mPa} \mathrm{s}$ ) and PP3 ( $\triangle$, blue; $\mu=1.90 \mathrm{mPa} \mathrm{s}$ ) with results for FC-72 ( $\diamond$, black; $\mu=0.64 \mathrm{mPa}$ ) from Ref. [22].

viscosity liquids would also follow the same master curve. Below we show that this is not the case.

Experimental.-In the present work we study the Leidenfrost vapor-layer drag reduction mechanism in four Flutec perfluorocarbon liquids (F2 Chemicals Ltd) with dynamic viscosities $\mu$ that vary nearly 25-fold: PP1 $\left(\rho=1.71 \mathrm{~g} / \mathrm{cm}^{3}, \quad \mu=0.81 \mathrm{mPa} \mathrm{s}\right), \quad$ PP3 $\quad\left(1.83 \mathrm{~g} / \mathrm{cm}^{3}\right.$, $1.90 \mathrm{mPa} \mathrm{s})$, PP10 (1.98 g/ $\left.\mathrm{cm}^{3}, 9.60 \mathrm{mPa} \mathrm{s}\right)$, and PP11 $\left(2.03 \mathrm{~g} / \mathrm{cm}^{3}, 19.2 \mathrm{mPa} \mathrm{s}\right)$. This range of viscosities allows the experimental Reynolds number to be varied over 2 orders of magnitude in contrast to previous experiments conducted with FC-72 (0.64 mPa.s) and $95{ }^{\circ} \mathrm{C}$ water (0.33 mPa.s). As we observe identical trends using all of the Flutec liquids, we will discuss in detail data for PP3 (1.90 mPa.s) and PP11 (19.2 mPa.s), and relegate results for PP1 and PP10 to the Supplemental Material [11]. Perfluorocarbon liquids are chosen for their purity, chemical inertness, thermal stability, and their moderate Leidenfrost temperatures. The experiments were conducted in a $1.6 \mathrm{~m}$ tall liquid tank with a $10 \times 10 \mathrm{~cm}$ cross section, using solid spheres of various materials heated to temperatures exceeding $T_{L}$. See Table $S 1$ in the Supplemental Material [11] for the physical properties including $T_{B}$ and $T_{L}$ of all liquids. The sphere trajectories were recorded using a high speed camera (Photron SA-5). The experimental protocol is similar to that in earlier work $[22,23]$ and further details can be found in the Supplemental Material [11].

Results.-Variations of the terminal velocity $U_{T}$ with the sphere temperature $T_{S}$ for PP3 and PP11 are presented in Fig. 1, together with reference data for FC-72. For each liquid the transition to the Leidenfrost regime is marked by a sharp increase in $U_{T}$ above $T_{L}$, reflecting the drag reduction by the development of a continuous vapor layer that envelopes the sphere. Experiments to determine $C_{D}$ vs Re were conducted using initial sphere temperatures $T_{S}=300^{\circ} \mathrm{C}$ in PP3 and $T_{S}=450{ }^{\circ} \mathrm{C}$ in PP11, different sphere sizes $(2 R=10,15,20 \mathrm{~mm})$, and sphere materials of different densities $\rho$ : sintered corundum $\left(3.8 \mathrm{~g} / \mathrm{cm}^{3}\right)$, steel $\left(7.8 \mathrm{~g} / \mathrm{cm}^{3}\right)$, and tungsten carbide $\left(14.9 \mathrm{~g} / \mathrm{cm}^{3}\right)$.

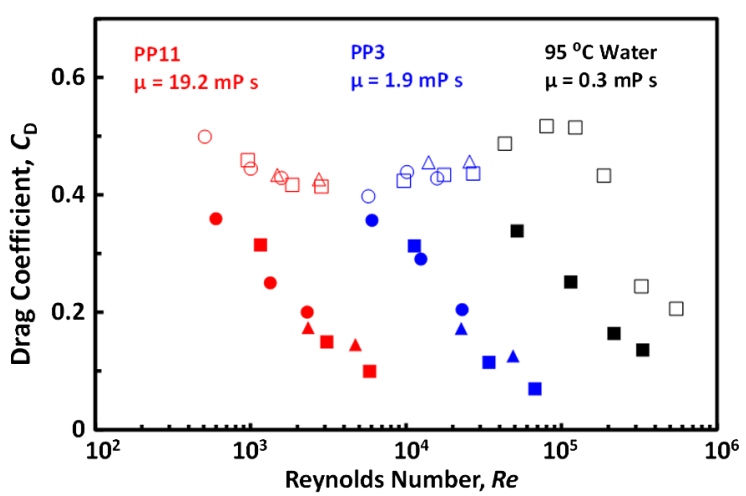

FIG. 2. Variation of the drag coefficient $C_{D}$ with Reynolds number for spheres free falling in PP11 $\left(3 \times 10^{2}<\operatorname{Re}<\right.$ $3 \times 10^{3}$, red $)$ and PP3 $\left(5 \times 10^{3}<\operatorname{Re}<8 \times 10^{4}\right.$, blue $)$. The open symbols are for different solid spheres at room temperature: steel $(\square, 2 R=10,15,20 \mathrm{~mm})$, sintered corundum $(\bigcirc, 2 R=10,15$, $20 \mathrm{~mm})$, and tungsten carbide $(\triangle, 2 R=10,15 \mathrm{~mm})$. The solid symbols correspond to spheres heated to $450{ }^{\circ} \mathrm{C}$ in PP11 or to $300{ }^{\circ} \mathrm{C}$ in PP3 before release to free fall in the Leidenfrost regime. The data for $95^{\circ} \mathrm{C}$ water $\left(\operatorname{Re}>4 \times 10^{4}\right.$, black) for steel spheres $(2 R=10,15,20$, or $25 \mathrm{~mm})$ without a vapor layer $(\square)$ and in the Leidenfrost regime (solid $\square$ ) are taken from Ref. [23]. See Fig. S3 in the Supplemental Material for an extended data collection including the PP1 and PP10 cases [11].

Results for the variation of the drag coefficient $C_{D}$ with Reynolds number Re for both PP11 and PP3 are presented in Fig. 2 together with data for $95{ }^{\circ} \mathrm{C}$ water, which is the lowest viscosity liquid studied. For each liquid we compare the corresponding case of spheres at room temperature without a vapor layer (open symbols) and the Leidenfrost regime case (solid symbols). To minimize clutter, we give results for the intermediate viscosity fluids PP1 and PP10 that follow the same trend in Fig. S3 in the Supplemental Material [11].

The common trend evident in Figs. 2 and S3 is that for all the liquids tested, the Leidenfrost vapor layer induces strong drag reduction reflected by the decrease of $C_{D}$ with increasing $\mathrm{Re}$, with a major departure from the standard drag curve. Note that the $C_{D}$ data for each separate liquid fall on their own master curve, when experiments for different sphere sizes and densities are plotted together. These curves have similar shapes, but are shifted to lower values of Re with increasing liquid viscosity.

The extent of the drag reduction is demonstrated most dramatically by comparing the free-fall trajectories of spheres in PP3 (video 1) and in PP11 (video 2) for (i) spheres at room temperature $T_{S}=22^{\circ} \mathrm{C}$, (ii) spheres at a temperature above the liquid's boiling temperature but below the Leidenfrost temperature, $T_{B}<T_{S}<T_{L}$, and (iii) spheres in the Leidenfrost regime $T_{S}>T_{L}$ [11]. In cases (ii) and (iii), the bubbles released from the sphere surface provide a visualization of the flow field in the wake. These videos also clearly demonstrate that the separation 
point of the wake on Leidenfrost spheres has moved towards the rear, thereby reducing the form drag. Although the delayed separation observed for the Leidenfrost spheres appears to be the same as the characteristic signature of the drag crisis transition on solid spheres, the present experiments indicate a very different dragreduction mechanism due to the presence of the vapor layer. For a smooth solid sphere, the delayed separation is due to the transition to turbulence in the viscous boundary layer at the critical $\operatorname{Re} \simeq 3 \times 10^{5}$. On the other hand, the Leidenfrost vapor layer can reduce the drag over a wide range of $\operatorname{Re}$, even down to $\operatorname{Re} \sim 600,3$ orders of magnitude smaller than for the conventional drag crisis. Therefore, it appears that the boundary-layer transition to turbulence plays no role in the mechanisms of the drag reduction by Leidenfrost vapor layers.

Modeling.-The vapor layer introduces new physical quantities that might affect the drag coefficient $C_{D}$. It is reasonable to assume the most important of these are the vapor layer properties, namely, its thickness $\lambda_{V}$, viscosity $\mu_{V}$, and density $\rho_{V}$, i.e., $C_{D}=f\left(\operatorname{Re}, \lambda_{V}, \mu_{V}, \rho_{V}\right)$. The drag force in the range of Re relevant here is dominated by the form drag in comparison to the viscous skin drag. The form drag depends primarily on the location of the separation point on the back side of the sphere. The location of the separation point is determined by the adverse pressure gradient that the flow encounters after crossing the equator and it occurs when the fluid elements next to the vapor layer decelerate sufficiently for the velocity gradient and shear stress to vanish. Relaxing the no-slip boundary condition at the sphere surface due to the vapor layer is expected to alter this boundary layer structure. Allowing for a partial-slip boundary condition is expected to move the separation point towards the rear stagnation point, as is the case for a rising bubble [34]. We propose to fit the effect of the vapor layer using the Navier slip model parametrized by a slip length [35].

We can estimate the thickness of the Leidenfrost vapor layer from the measured rate of cooling $d T_{S} / d t$ of a hot sphere in the Leidenfrost regime using the relation $\lambda_{V}=$ $\left(3 k_{v} / \rho_{S} c_{p} R\right)\left(T_{S}-T_{\text {sat }}\right) /\left(d T_{S} / d t\right)[23,36]$, where $\rho_{S}$ is the sphere density, $c_{p}$ is the sphere specific heat, $k_{v}$ is the vapor thermal conductivity, and $T_{\text {sat }}$ is the saturation temperature of the liquid. We find $\lambda_{V} \sim 150 \pm 50 \mu \mathrm{m}$ for all of the perfluorocarbon fluids studied here (see the details on p. 4 of the Supplemental Material and Fig. S2 [11]). In reality, the thickness of the Leidenfrost vapor layer, $\lambda_{V}$ on the sphere is not uniform, so rather than attempting to model the detailed flow structures associated with a dynamic vapor layer of nonuniform thickness, we parametrize our experimental observations using the Navier slip model. Here, the familiar no-slip boundary condition at the surface of the sphere is replaced by the condition that the tangential velocity is proportional to the tangential stress at the surface, with the constant of proportionality being the slip length $\lambda_{S}[24,25,29,35]$.

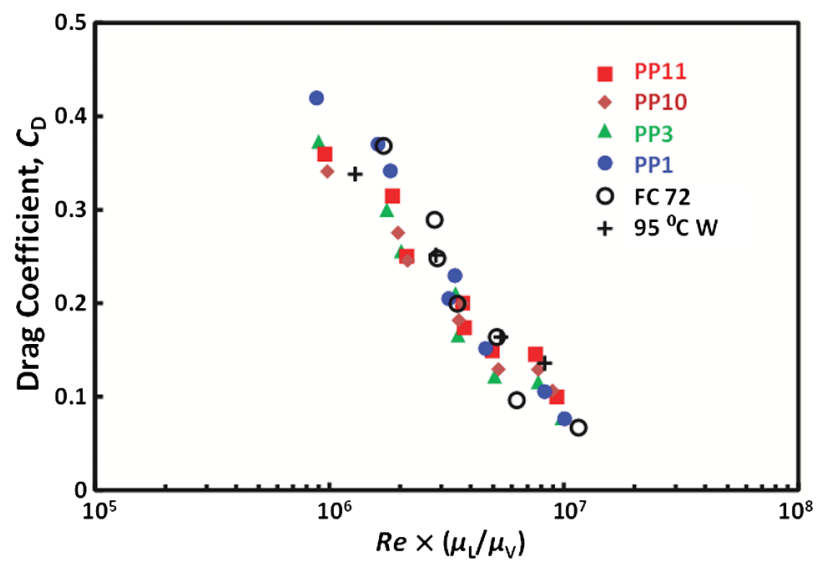

FIG. 3. Dependence of the drag coefficient $C_{D}$ on the Reynolds number multiplied by the liquid to vapor viscosity ratio, $\operatorname{Re} \times\left(\mu_{L} / \mu_{V}\right)$, for various spheres free falling in the Leidenfrost regime in the various liquids studied: PP11 (solid squares, red), PP10 (solid diamonds, brown), PP3 (solid triangles, green), PP1 (solid circles, blue), FC-72 (open circles, black) [22], and $95{ }^{\circ} \mathrm{C}$ water (crosses, black) [23].

For $\operatorname{Re} \sim 0$, the effect of a uniform vapor layer of thickness $\lambda_{V}$ can be mapped to a Navier slip length $\lambda_{S}$ by

$$
\lambda_{S} \sim\left(\mu_{L} / \mu_{V}\right) \lambda_{V}
$$

with a constant of proportionality that is a function of the problem geometry $[25,29,30]$. Thus, for the fluorocarbon liquids studied here, where $\mu_{L} / \mu_{V}$ varies from $\sim 70$ for PP1 to 1900 for PP11 [11], the slip length is expected to increase with the liquid viscosity $\mu_{L}$ and to be orders of magnitude larger than the vapor layer thickness.

At the high Re regime, investigated here, there is no quantitative theory that can relate $\lambda_{S}$ to $\lambda_{V}$. However, we assume that the trend for the slip length to increase with liquid viscosity captured in relation (1) will be preserved. We further note that the master curves in Figs. 2 and $3 \mathrm{~S}$ collapse using the simplest scaling $C_{D} \sim f\left(\operatorname{Re}, \lambda_{S}\right) \sim \operatorname{Re} \times$ $\left(\mu_{L} / \mu_{V}\right)$, as $\lambda_{V}$ is approximately constant for our liquids. This is shown in Fig. 3. We do not, however, expect this empirical collapse to be universal, as $\lambda_{V}$ can change with different liquid-vapor thermodynamic properties.

To test this further, a series of direct numerical simulations (DNS) and large eddy simulations (LES) were undertaken using the commercial finite-volume package FLUENT 15.0, with a Navier slip boundary condition implemented at the sphere surface. The DNS was performed for $\operatorname{Re}<10^{4}$ in which case the flow field around the sphere remains laminar, and the LES was performed for $\operatorname{Re}>10^{4}$ using a dynamic Smagorinsky turbulence model, consistent with previous studies [37-39]. For zero slip length, we verified that our calculations based on this model can reproduce all known literature computational and experimental variations of the drag coefficient and wake separation angle (see Fig. S5 [11]). Details of the model, 


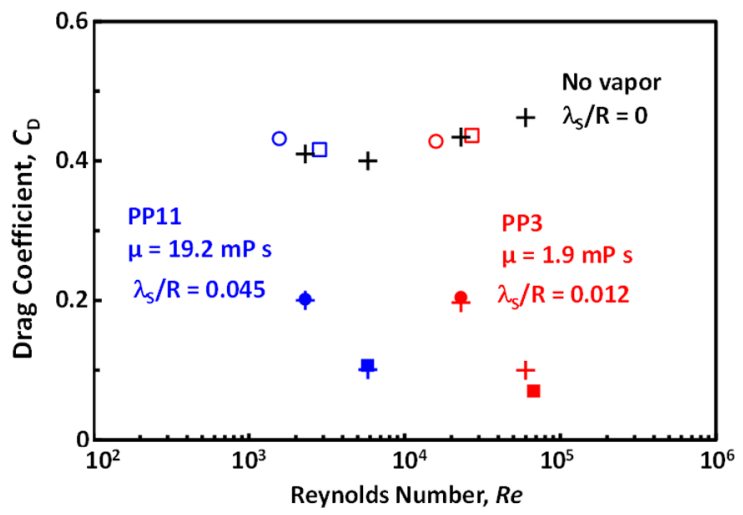

FIG. 4. Comparison of the Reynolds number dependence of the drag coefficients $C_{D}$ measured in PP11 ( Re $<10^{4}$, blue) and in PP3 $\left(\operatorname{Re}>10^{4}\right.$, red) with the numerical simulation results $(+)$. Experimental results are for a $2 R=20 \mathrm{~mm}$ steel $(\square)$ or $2 R=$ $20 \mathrm{~mm}$ sintered corundum $(O)$ sphere in the presence (solid symbols) and absence (open symbols) of a Leidenfrost vapor layer (Fig. 2). The simulation results $(+)$ were obtained using slip lengths of $\lambda_{S} / R=0$ (black) for the no vapor layer case, and $\lambda_{S} / R=0.045$ (blue) for the PP11 and $\lambda_{S} / R=0.012$ (red) for the PP3 Leidenfrost vapor layer case.

mesh information, and validation are available in the Supplemental Material [11].

In Fig. 4, we compare the measured values of $C_{D}$ to those obtained from the simulations, for a selection of PP3 and PP11 data points. For no slip, the $\lambda_{S}=0$ simulation results and the no vapor layer experimental data are in good agreement with the classical $C_{D}(\mathrm{Re})$ curve. In the presence of the Leidenfrost vapor layer, the $C_{D}$ vs Re dependence in both liquids is well captured by the Navier slip model, with a slip length scaled by the sphere radius, $\lambda_{S} / R=0.045$ for PP11 and $\lambda_{S} / R=0.012$ for PP3. Thus, $\lambda_{S} / R$ in the simulations increases by a factor 4 when $\mu_{L}$ increases tenfold, following the trend anticipated from the experimental results. Also, we see in Fig. 5 and in video 3 [11] that the Navier slip model is capable of reproducing the shape of the wakes and the point of separation of the laminar boundary layer associated with the drag reduction due to the presence of the vapor layer.

The use of the Navier slip length that is assumed to be constant over the surface of the falling sphere is a simplified model of a dynamic Leidenfrost vapor layer of nonuniform thickness. Indeed, the same comment can be applied to the use of uniform concentric vapor layer models to represent entrained surface air layers or plastrons [29-31]. However, given the technical challenges of modeling high Re flows, the simple Navier slip model perhaps performed unexpectedly well in being able to also predict the change in the flow pattern associated with the onset of drag reduction. At the same time we recognize that the slip length only serves as a convenient parametrization of an idealized model and caution against the over interpretation or assignment of an actual physical attribute associated with the slip length.
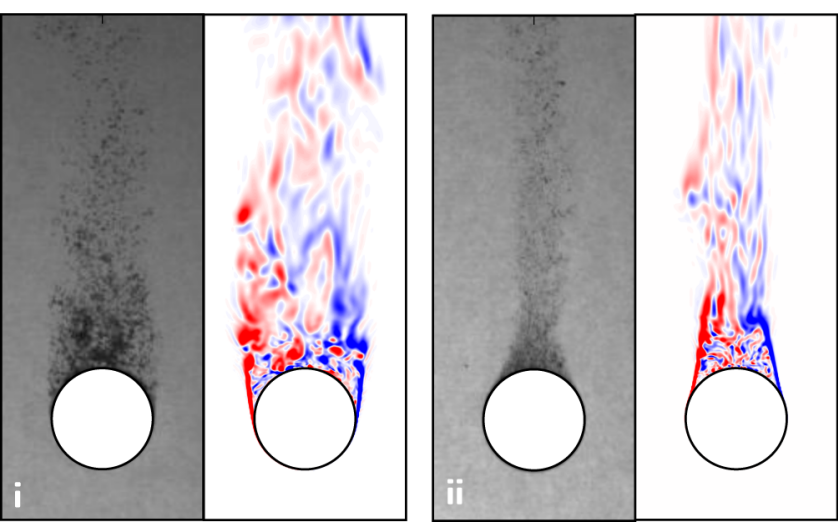

FIG. 5. Comparison of instantaneous wakes for experiment and simulation showing contours of instantaneous out-of-plane vorticity: i) No vapour layer, $\mathrm{Re}=2.7 \times 10^{4}$ (left) and $\lambda_{S} / R=0$, $\mathrm{Re}=2.3 \times 10^{4}$ (right), ii) Leidenfrost vapor layer, $\mathrm{Re}=6.7 \times$ $10^{4}$ (left) and $\lambda_{S} / R=0.012, \operatorname{Re}=6 \times 10^{4}$ (right). See also Video 3 and Supplemental Information [11] for details of the numerical and experimental visualisation.

Conclusions.—By a systematic experimental study using a series of pure and inert fluorocarbon liquids, we have uncovered a drag reduction phenomenon arising from a Leidenfrost vapor layer that envelopes a bluff body. The observed drag reduction spans 3 orders of magnitude of Re as the liquid viscosity $\mu_{L}$ is varied almost 25 -fold, thus experimentally establishing a hypothesized vapor layer drag reduction mechanism that far exceeds the range of other drag reduction methods. The variation of $C_{D}$ vs Re for a Leidenfrost sphere in free fall can be fitted quantitatively by DNS and LES with the Navier slip boundary condition to account for the presence of a vapor layer. As anticipated with the low Re result, larger liquid viscosities correlate with a higher effective slip length. The model also predicted correctly the abrupt change in the wake pattern associated with the onset of drag reduction owing to the vapor layer. This is a preliminary attempt to provide a semiquantitative fit to the observed experimental variation of $C_{D}$ vs Re. More sophisticated considerations in modeling transitional flows, such as the subgrid stresses in the Smagorinsky model that might modify the laminar flow and perhaps influence the boundary layer separation and modify the drag-reduction mechanism, remain open questions to be explored.

This work was supported by the King Abdullah University of Science and Technology and in part by the Australian Research Council through a Discovery Project Grant to D. Y. C. C.

*ivanuriev.vakarelski@kaust.edu.sa

[1] E. Achenbach, J. Fluid Mech. 54, 565 (1972).

[2] H. Choi, W-P. Jeon, and J. Kim, Annu. Rev. Fluid Mech. 40, 113 (2008). 
[3] S. Hong and T. Asai, Sci. Rep. 4, 5068 (2014).

[4] C. Clanet, Annu. Rev. Fluid Mech. 47, 455 (2015).

[5] A. White, Nature (London) 216, 994 (1967).

[6] S. Jeon, J. Choi, W.-P. Jeon, H. Choi, and J. Park, J. Fluid Mech. 517, 113 (2004).

[7] I. U. Vakarelski, D. Y. C. Chan, and S. T. Thoroddsen, Phys. Rev. Lett. 115, 044501 (2015).

[8] J. G. Leidenfrost, De Aquae Communis Nonnullis Qualitatibus Tractatus (Ovenius, Duisburg ad Rhenum, 1756) [Int. J. Heat Mass Transfer 9, 1153 (1966)].

[9] I. U. Vakarelski, N. A. Patankar, J. O. Marston, D. Y. C. Chan, and S. T. Thoroddsen, Nature (London) 489, 274 (2012).

[10] D. Quéré, Annu. Rev. Fluid Mech. 45, 197 (2013).

[11] See Supplemental Material at http://link.aps.org/ supplemental/10.1103/PhysRevLett.117.114503 for, which includes Refs. [12-21] and Figs. S1-S5, for experimental and numerical simulation details.

[12] H. Lamb, Hydrodynamics (Dover Publications, New York, 1932). This is the first reference in Ref. [11] not already in the Letter.

[13] D. K. Lilly, Phys. Fluids A 4, 633 (1992).

[14] D. A. Jones and D. B. Clarke, Report No. DSTO-TR-2232, 2008 (unpublished).

[15] E. K. W. Poon, G. Iaccarino, A. S. H. Ooi, and M. Giacobello, Numerical studies of high Reynolds number flow past a stationary and rotating sphere, in Proceedings of 7th International Conference of CFD in the Minerals and Process Industries (CSIRO, Melbourne, Australia, 2009).

[16] S. Jindal, L. Long, P. Plassmann, and N. Uzol, Large Eddy Simulations around a Sphere Using Unstructured Grid, in Proceedings of 34th AIAA Fluid Dynamics Conference, paper 2228 (AIAA, Reston, VA, 2004).

[17] R. Clift, J. Grace, and M. Weber, Bubbles, Drops, and Particles, (Academic Press, New York, 1978).

[18] I. Rodriguez, R. Borell, O. Lehmkuhl, C. D. Perez-Segarra, and A. Oliva, J. Fluid Mech. 679, 263 (2011).

[19] V. Seidl, S. Muzaferija, and M. Peric, Appl. Sci. Res. 59, 379 (1997).
[20] A. G. Tomboulides, Ph.D. thesis, Princeton University (1993).

[21] P. Ploumhans, G. S. Winckelmans, J. K. Salmon, A. Leonard, and M.S. Warren, J. Comput. Phys. 178, 427 (2002). This is the last reference in Ref. [11] not already in the Letter.

[22] I. U. Vakarelski, J. O. Marston, D. Y. C. Chan, and S. T. Thoroddsen, Phys. Rev. Lett. 106, 214501 (2011).

[23] I. U. Vakarelski, D. Y. C. Chan, and S. T. Thoroddsen, Soft Matter 10, 5662 (2014).

[24] C. Cottin-Bizonne, J.-L. Barrat, L. Bocquet, and E. Charlaix, Nat. Mater. 2, 237 (2003).

[25] D. C. Tretheway and C. D. Meinhart, Phys. Fluids 16, 1509 (2004).

[26] J. P. Rothstein, Annu. Rev. Fluid Mech. 42, 89 (2010).

[27] S. L. Ceccio, Annu. Rev. Fluid Mech. 42, 183 (2010).

[28] G. McHale, M. I. Newton, and N. J. Shirtcliffe, Soft Matter 6, 714 (2010).

[29] G. McHale, M. R. Flynn, and M. I. Newton, Soft Matter 7, 10100 (2011).

[30] A. Busse, N. D. Sandham, G. McHale, and M. I. Newton, J. Fluid Mech. 727, 488 (2013).

[31] B. R. K. Gruncell, N. D. Sandham, and G. McHale, Phys. Fluids 25, 043601 (2013).

[32] C. Barbier, E. Jenner, and B. D’Urso, arXiv:1406.0787.

[33] J. C. Brennan, N. R. Geraldi, R. H. Morris, D. J. Fairhurst, G. McHale, and M. I. Newton, Sci. Rep. 5, 10267 (2015).

[34] D. W. Moore, J. Fluid Mech. 16, 161 (1963).

[35] J. W. Swan and A.S. Khair, J. Fluid Mech. 606, 115 (2008).

[36] H. I. Jouhara and B. P. Axcell, Trans. I Chem E, Part A (Chem. Eng. Res. Design) 80, 284 (2002).

[37] M. Germano, U. Piomelli, P. Moin, and W. H. Cabot, Phys. Fluids A 3, 1760 (1991).

[38] G. S. Constantinescu and K. D. Squires, Flow Turbul. Combust. 70, 267 (2003).

[39] G. Constantinescu and K. Squires, Phys. Fluids 16, 1449 (2004). 